\title{
A Family of Codes between Some Markov and Bernoulli Schemes
}

\author{
Gabriella Monroy and Lucio Russo \\ Istituto di Fisica Teorica, Università di Napoli, Napoli, Italy
}

Received February 13, 1975

\begin{abstract}
We construct a family of almost continuous codes between a mixing one-step Markov process with two symbols and a Bernoulli scheme.
\end{abstract}

\section{Introduction}

It is known (see Ref. [2]) that two "finitely determined" processes with the same entropy are isomorphic. In particular this statement implies that a mixing Markov chain is isomorphic to a Bernoulli scheme with the same entropy $([1,2])$. The isomorphism means, in the language of codings, that one can find invertible and shift-invariant maps between the typical sequences of the two processes. Such "codes", nevertheless, are explicitely known only in a few cases (see [4]); moreover, it is not known whether, endowing the space of sequences with the natural topology defined below, it is possible to construct almost continuous codes.

Here we construct explicitely an uncountable family of almost continuous invertible codes between a mixing one-step Markov process with two symbols and a Bernoulli scheme.

\section{Symbols and Definitions}

Let $X=\{-1,1\}^{\mathbb{Z}}$ be the set of all doubly infinite sequences of the two numbers $-1,1, \mathscr{B}$ the $\sigma$-algebra of the Borel sets in $X$ (endowed with the topology obtained as product of the discrete topologies on the factors); let $T: X \rightarrow X$ be the map defined by:

$$
(T x)_{i}=x_{i+1} .
$$

If $\mu_{S}$ is a $T$-invariant measure defined on $\mathscr{B}$, we call the triple $S=\left(X, T, \mu_{S}\right)$ a "shift". If, moreover, $\varphi: X \rightarrow X$ is a Borel map commuting with $T$, let us define:

$$
\bar{\varphi} S=\left(X, T, \bar{\varphi} \mu_{S}\right), \quad \text { with } \quad \bar{\varphi} \mu_{S}(Y)=\mu_{S}\left(\varphi^{-1} Y\right) \quad \forall Y \in \mathscr{B} .
$$

Let $0<\alpha<1$; call $B_{\alpha}$ the (Bernoulli) shift whose measure $\mu_{B_{\alpha}}$, extended to $\mathscr{B}$ by Kolmogorov's theorem, is defined on the cylinders of $X$ by:

$$
\mu_{B_{\alpha}}\left(\left\{x \in X \mid x_{i+1}=k_{1} \ldots x_{i+l}=k_{l}\right\}\right)=\prod_{\substack{1 \leqq r \leqq l \\ k_{r}=1}} \alpha \prod_{\substack{1 \leqq r \leqq l \\ k_{r}=-1}}(1-\alpha) .
$$


In a similar way we can define the one-step Markov shift $M_{\alpha}$ by letting:

$$
\mu_{M_{\alpha}}\left(\left\{x \in X \mid x_{i+1}=k_{1} \ldots x_{i+l}=k_{l}\right\}\right)=\frac{1}{2} \prod_{\substack{1<r \leq l \\ k_{r}=k_{r-1}}} \alpha \prod_{\substack{1<r \leq l \\ k_{r} \neq k_{r-1}}}(1-\alpha) .
$$

It is easy to check that $B_{\alpha}$ and $M_{\alpha}$ have the same entropy, and thus they are (Refs. $[1,3])$ isomorphic. This implies the existence of an invertible measurable map $\psi: X \rightarrow X$ commuting with $T$ defined $\mu_{M_{\alpha}}$-a.e., such that:

$$
\bar{\psi} M_{\alpha}=B_{\alpha} .
$$

Using the definitions (2.2) and (2.3) one can immediately check that the map $\varphi$ defined by letting:

$$
(\varphi x)_{i}=x_{i-1} \cdot x_{i}
$$

satisfies (2.4); on the other hand the map $\varphi$ is clearly not-invertible. It is known, however, that, if we introduce in the set of the measurable mappings commuting with $T$ the metric:

$$
\varrho(\varphi, \psi)=\mu_{M_{\alpha}}\left(\left\{x \in X \mid(\varphi x)_{0} \neq(\psi x)_{0}\right\}\right) .
$$

$\varphi$ is an accumulation point of invertible mappings verifying (2.4) (see Ref. [2], Part I, Proposition 11 of Section 4).

In the next section, in effect, we construct an uncountable family of such mappings by modifying slightly $\varphi$.

\section{The Code}

Let $M=\left\{M_{i}\right\}_{i=1,2 \ldots}$ be a strictly increasing sequence of positive integers, with $M_{1} \geqq 3$, and let $\xi \in X$. We call " $n$-string" of $\xi$ each $k$-ple of consecutive indices of $\xi,[i+1, \ldots, i+k]$ such that:

$$
\xi_{i+1}=\cdots \xi_{i+k}=1 ; \quad \xi_{i}=\xi_{i+k+1}=-1 ; \quad M_{n} \leqq k<M_{n+1} .
$$

Then we call the set $[i+1, \ldots, i+k]$ an " $n$-block" of $\xi$ if it is contained between two consecutive $n$-strings (i.e. $i$ is the endpoint of an $n$-string and $i+k+1$ is the starting point of the following one) and there are no strings of higher order contained in it.

Moreover, we call "changeable" an element $\xi_{i}$ of $\xi$ if by changing its sign the positions and the lenghts of blocks and strings remain unaltered.

Let us consider, now, an $n$-block $B=[i+1, \ldots, i+k]$ of $\xi$ and let $l \leqq k / 2$, be the least positive integer (if it exists) such that:

1) $\xi_{i+1+l} \neq \xi_{i+k-l}$.

2) In the segments $[i+1, \ldots, i+l]$ and $[i+k-l, \ldots, i+k]$ there are not strings.

3) $\xi_{i+1+l}$ and $\xi_{i+k-l}$ are "changeable".

If it is possible to find such an $l$, we call "conjugate elements associated to $B$ " the pair $\left\{\xi_{i+1+l}, \xi_{i+k-l}\right\}$. It is clear that an element $\xi_{i}$ can belong to at most one such pair. 
It is not difficult to check that, $\mu_{B_{\alpha}}$ - a.e., each element of a sequence $\xi$ belongs to infinitely many blocks and is between infinitely many pairs of conjugate elements.

We can now define, $\mu_{M_{\alpha}}-$ a.e., the map $\psi_{M}: X \rightarrow X$ in the following way:

If $(\varphi x)_{i}$ does not belong to a pair of conjugate elements of the sequence $\varphi x$, we put:

$$
\left(\psi_{M} x\right)_{i}=(\varphi x)_{i}
$$

If, instead, $(\varphi x)_{i}$ is one of the two conjugate elements associated to a block $B$ of $\varphi x$, we put:

$$
\left(\psi_{M} x\right)_{i}=(\varphi x)_{i} \cdot x_{n_{B}}
$$

where $n_{B}$ is the first element of the block $B$. In other words, the sequence $\psi_{M} x$ is obtained from the sequence $\varphi x$ by changing only the conjugate elements associated to the blocks (of $\varphi x$ ) that "in the sequence $x$ begin with -1 " (note that it is surely $\left.(\varphi x)_{n_{B}}=-1\right)$.

Remark 1. Note that the sequences $\varphi x$ and $\psi_{M} x$ have blocks, strings and conjugate elements in the same positions.

Remark 2. If we denote $-x$ the sequence defined by $(-x)_{i}=-x_{i}$ and we call $\sigma_{M} \xi$ the sequence obtained from $\xi$ by changing the sign of all the conjugate elements of $\xi$, we have:

$$
\varphi(-x)=\varphi(x) ; \quad \psi_{M}(-x) \approx \sigma_{M} \psi_{M}(x) .
$$

\section{Main Results}

We collect in the following proposition some statements which easily follow from the construction of $\psi_{M}$ :

Proposition 1. For every increasing sequence $M$ of positive integers with $M_{1} \geqq 2$ the map $\psi_{M}$ commutes with $T$, is measurable with respect to $\mathscr{B}$ and is $\mu_{M_{\alpha}}$-almost continuous. If $M$ and $M^{\prime}$ are two different sequences:

$$
\mu_{M_{\alpha}}\left(\left\{x \in X \mid\left(\psi_{M} x\right)_{0} \neq\left(\psi_{M^{\prime}} x\right)_{0}\right\}\right)>0 .
$$

Hence by varying $M$ we obtain an uncountable family of essentially different mappings.

Theorem 1. For every $M, \psi_{M}$ is $\mu_{M_{\alpha}}$-a.e. invertible and its inverse is almost continuous.

Proof. We shall show that for $\mu_{B_{\alpha}}$-almost every $\xi \in X$, there is a unique $x \in X$ such that:

$$
\psi_{M} x=\xi .
$$

Let $\xi \in X$; for every index $k$, let $B(k)$ be the least-order block of $\xi$ such that:

1) $k$ belongs to the block $B(k)$.

2) There are, associated to $B(k)$, two conjugate elements, $i, j$, such that $i \leqq k<j$. 
It is clear that for a set of $\mu_{B_{\alpha}}$-measure 1 such a block exists. Let $\alpha(k)$ be the first element of $B(k)$ [note that 2) implies that $k$ is at least the second element of $B(k)]$.

We observe that, for every sequence $x$ :

$$
x_{k}=x_{\alpha(k)} \prod_{l=\alpha(k)+1}^{k}(\varphi x)_{l} .
$$

Suppose now that $x$ satisfies (4.1). If $l$ is not a conjugate element of $\xi$ by Remark 1 $l$ cannot be a conjugate element of $\varphi x$ and we have $(\varphi x)_{l}=\xi_{l}$; if $r, s$ are two conjugate elements of $\xi$ we get

$$
(\varphi x)_{r}(\varphi x)_{s}=\xi_{r} \xi_{s} .
$$

Moreover the only conjugate element which occurs alone in the segment $[\alpha(k), k]$ is $i$. Hence:

$$
\prod_{l=\alpha(k)+1}^{k}(\varphi x)_{l}=\prod_{\substack{\alpha(k)+1 \leqq l \leqq k \\ l \neq i}} \xi_{l}(\varphi x)_{i} .
$$

On the other hand, the construction of $\psi_{M}$ implies that:

$$
x_{\alpha(k)}(\varphi x)_{i}=\xi_{i} .
$$

Using the last two equalities and (4.2) we have:

$$
x_{k}=\prod_{l=\alpha(k)+1}^{k} \xi_{l} \text {. }
$$

We have proved that the only sequence which can satisfy (4.1) is the one defined in (4.3). To verify that this sequence actually satisfies (4.1) note that

if and only if:

$$
(\varphi x)_{k}=-\xi_{k}
$$

If we put:

$$
B(k-1) \neq B(k) ; \prod_{l=\alpha(k-1)+1}^{k-1} \xi_{l}=-\prod_{l=\alpha(k)+1}^{k-1} \xi_{l}
$$

(4.4) means:

$$
\begin{aligned}
& \alpha=\min [\alpha(k-1), \alpha(k)], \\
& \beta=\operatorname{Max}[\alpha(k-1), \alpha(k)]
\end{aligned}
$$

$$
B(k) \neq B(k-1) ; \prod_{k=\alpha+1}^{\beta} \xi_{k}=-1 .
$$

This occurs if and only if $k$ is a conjugate element of $\xi$ associated to a block starting in a point $\beta$ such that $x_{\beta}=-1$. Furthermore the conjugate elements of $\xi$ and $\varphi(x)$ are the same.

Hence the sequence defined in (4.3) satisfies (4.1).

The last part of the theorem easily follows from the construction of $\psi_{M}^{-1}$.

Theorem 2. For every $M, \bar{\psi}_{M} M_{\alpha}=B_{\alpha}$. 
Proof. We take into account the finite sequences of the two numbers $-1,1$ of the type $\xi_{i} f_{1} B f_{2} \xi_{l}$, where $B$ is an $n$-block, $f_{1}$ and $f_{2}$ are $n$-strings and $\xi_{i}, \xi_{l}$ are two negative elements. Let $F$ be such a sequence and $C_{F}$ the corresponding cylinder:

$$
C_{F}=\left\{\xi \in X \mid \xi_{1} \ldots \xi_{r}=\xi_{i} f_{1} B f_{2} \xi_{l}\right\}
$$

One can easily realize that

$$
\psi_{M}^{-1}\left(C_{F}\right)=C_{G} \cup C_{\tilde{G}},
$$

where $G, \tilde{G}$ are finite sequences such that

We have

$$
\begin{aligned}
\varphi\left(C_{\tilde{G}}\right) & =\sigma_{M} \varphi\left(C_{G}\right) ;(\tilde{G})_{i}=-G_{i} \\
\mu_{B_{\alpha}}\left(C_{F}\right) & =\mu_{B_{\alpha}}\left(C_{\varphi G}\right) .
\end{aligned}
$$

$$
\begin{aligned}
\mu_{M_{\alpha}}\left(C_{G}\right) & =\frac{1}{2} \mu_{B_{\alpha}}\left(C_{\varphi G}\right) \\
\mu_{M_{\alpha}}\left(\psi_{M}^{-1} C_{F}\right) & =\mu_{M_{\alpha}}\left(C_{G}\right)+\mu_{M_{\alpha}}\left(C_{\tilde{G}}\right) \\
& =\mu_{B_{\alpha}}\left(C_{\varphi G}\right)=\mu_{B_{\alpha}}\left(C_{F}\right) .
\end{aligned}
$$

Since the cylinders of the type $C_{F}$, with their translates, generate the $\sigma$-algebra $\mathscr{B}$, we have

$$
\mu_{M_{\alpha}}\left(\psi_{M}^{-1} Y\right)=\mu_{B_{\alpha}}(Y) \quad \forall Y \in \mathscr{B} .
$$

Acknowledgements. We are greatly indebted to G. Gallavotti for having proposed the problem and for very useful discussions of the results undertaken with us during all the completion of the work. Many discussions with F. di Liberto and R. Esposito are also acknowledged.

\section{References}

1. Friedman, N.A., Ornstein, D. S. : Adv. Math. 5, 365 (1971)

2. Ornstein, D. S. : Ergodic theory, randomness and dynamical systems. Yale: University Press 1974

3. Smorodinsky, M.: Ergodic theory, entropy. In: Lecture Notes in Mathematics. Berlin-HeidelbergNew York: Springer 1971

4. Jacobs, K.: Lecture notes on ergodic theory. University of Aarhus, Denmark (1962_1963)

Communicated by G. Gallavotti

Gabriella Monroy

Lucio Russo

Università di Napoli

Istituto di Fisica Teorica

Mostra d'Oltremare

Pad. 19

I-80125 Napoli, Italy 
